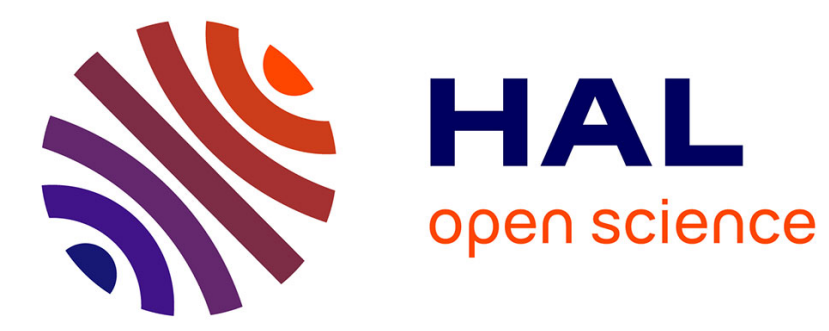

\title{
Collapse of a polymer chain in a melt of incompatible polymer chains
}

\author{
J.-F. Joanny, F. Brochard
}

\section{To cite this version:}

J.-F. Joanny, F. Brochard. Collapse of a polymer chain in a melt of incompatible polymer chains. Journal de Physique, 1981, 42 (8), pp.1145-1150. 10.1051/jphys:019810042080114500 . jpa-00209101

\section{HAL Id: jpa-00209101 https://hal.science/jpa-00209101}

Submitted on 1 Jan 1981

HAL is a multi-disciplinary open access archive for the deposit and dissemination of scientific research documents, whether they are published or not. The documents may come from teaching and research institutions in France or abroad, or from public or private research centers.
L'archive ouverte pluridisciplinaire HAL, est destinée au dépôt et à la diffusion de documents scientifiques de niveau recherche, publiés ou non, émanant des établissements d'enseignement et de recherche français ou étrangers, des laboratoires publics ou privés. 


\title{
Collapse of a polymer chain in a melt of incompatible polymer chains
}

\author{
J. F. Joanny and F. Brochard \\ Physique de la Matière Condensée $\left({ }^{*}\right)$, Collège de France, 11, place Marcelin-Berthelot, 75231 Paris cedex 05, France
}

(Reçu le 9 mars 1981, accepté le 8 avril 1981)

\begin{abstract}
Résumé. - On étudie la conformation d'une chaîne polymérique A dans un fondu de chaînes polymériques $B$ de nature chimique différente dans le domaine de température où il y a incompatibilité entre les chaînes $\mathrm{A}$ et $\mathrm{B}$. On est conduit à une forte contraction de la chaîne $\mathrm{A}$ assez similaire à l'effondrement d'une chaîne dans un mauvais solvant. La transition entre l'état idéal et l'état effondré est plus abrupte que pour une chaîne dans un mauvais solvant.
\end{abstract}

\begin{abstract}
We study the conformation of a linear polymer chain A in a melt of linear polymeric chains B of a different chemical species in the temperature domain where A and B chains are incompatible. We are lead to a strong contraction of the A chain similar to the usual collapse of a single chain in a poor solvent. However, the transition between the ideal and the collapsed state of the coils is more abrupt than for a single chain in a poor solvent.
\end{abstract}

1. Introduction. - Although we find in the literature a very large number of studies both theoretical and experimental on the thermodynamic properties of flexible polymer chains in a poor solvent (below the compensation temperature $\theta)[1,2,4]$, very little work has been devoted to the study of incompatible polymer melts. The reason is that these big objects usually show a very strong incompatibility, and at room temperature, such melts separate into two phases, one composed mostly of one polymer, the other one of the other.

Recently however, some cases of quasi-compatibility have been proposed [3] (melts of deuterated and hydrogenated chains or melts of homopolymers and random copolymers) and one can hope that in the near future there will be more experimental studies on these systems.

In this note, we study the collapse of a single $\mathrm{A}$ chain of $N$ monomers in a melt of B chains each of them having $P$ monomers. When the interaction energy between a monomer A and a monomer B is positive and large, we are lead to a strong effective attraction between the A monomers and the A chain collapses on itself.

\footnotetext{
(*) Equipe de recherche associée au C.N.R.S. no 542.
}

LE JOURNAL DE PHYSIQUe - T. 42, № 8, AOÛT 1981
The problem of one single polymer chain in a poor solvent $(T<\theta)$ which corresponds to the limit $P=1$ has been extensively studied both theoretically [8] and experimentally $[10,11]$. If the temperature of the solution is close to the Flory compensation temperature $\left(\frac{\theta-T}{\theta}<N^{-1 / 2}\right)$ the chain is ideal. Below such temperatures, we reach a collapsed state where the radius of gyration $R$ scales like $N^{1 / 3}\left(\frac{\theta}{\theta-T}\right)^{1 / 3}$. The cross-over between these two temperature regimes is very sharp, which leads several authors to talk about a "coil-globule transition", the coil being the ideal state, the globule the collapsed state.

Our discussion might be relevant for the problem of block copolymer surfactants in a melt, studied by Cantor [5]. The two parts of the block copolymers being highly incompatible, one of them could collapse in a microphase composed mostly of the other one.

2. Collapse of a long chain among shorter chains. In the appendix, we show by a self-consistent field approach that the free energy of one A chain of $N$ monomers among B chains each of them containing $P$ monomers as a function of the concentration $c$ of $\mathrm{A}$ monomers is 


$$
F=\int \mathrm{d}^{3} r\left\{\frac{k T a^{5}}{24}\left[\frac{1}{c a^{3}}+\frac{1}{1-c a^{3}}\right](\nabla c)^{2}+\frac{k T}{P a^{3}}\left(1-c a^{3}\right) \log \left(1-c a^{3}\right)+U_{\mathrm{int}}(c)\right\}
$$

and that the concentration $c$ varies over a length

$$
e=a\left(\frac{1}{c a^{3}}+\frac{1}{1-c a^{3}}\right)^{1 / 2}\left(\frac{P}{|\tau|}\right)^{1 / 2}
$$

$a$ is the step length of the monomer and $U_{\text {int }}(c)$ an interaction energy which, following Flory [1], can be written as :

$$
U_{\mathrm{int}}=\chi k T c\left(1-c a^{3}\right)+\sum_{i>3} A_{i} c^{(i)} .
$$

It is convenient to represent the temperature parametrically through $\tau=1-2 \chi P$.

If we suppose that $e$ is smaller than the radius $R$ of the A chains, the first term can be interpreted as a confinement energy. We can perform the integration with the approximation $\nabla c=\frac{c}{e}$ on a slice of size $e$ around the A chain, $\nabla c=0$ everywhere else, and $c=\frac{N}{\frac{4 \pi R^{3}}{3}}$. We obtain the free energy of the A chain

$$
\frac{F}{k T}=\frac{3}{2} \frac{1}{1-c a^{3}} \frac{N a^{2}}{R e}+\frac{N}{\left(a^{3} c\right) P}\left(1-c a^{3}\right) \log \left(1-c a^{3}\right)+\frac{N}{c a^{3}} \frac{U_{\mathrm{int}}(c)}{k T} .
$$

When the thickness of the boundary layer $e$ becomes equal to the radius of the A chain, the concentration of $\mathrm{A}$ monomers varies all over the radius of the $\mathrm{A}$ chain and one may approximate $\nabla c$ by $c / R$; in that approximation one usually speaks of a confinement free energy for the gradient term in (1). In that approximation

$$
\frac{F}{k T}=\frac{3}{2} \frac{1}{1-c a^{3}} \frac{N a^{2}}{R^{2}}+\frac{N}{\left(a^{3} c\right) P}\left(1-c a^{3}\right) \log \left(1-c a^{3}\right)+\frac{N}{c a^{3}} \frac{U_{\text {int }}(c)}{k T} .
$$

In fact, the self-consistent field approach in the ground state dominance approximation always eliminates the unperturbed ideal chain behaviour. That is the reason why the Flory elastic term, $3 R^{2} / 2 N a^{2}$, which usually appears, is absent in the free energy expressions ; e.g. (3) or (4). In order to study the radius of gyration for negative $\tau$, we will add such a term in the free energy. That elastic energy tends to bring the chain back to its ideal behaviour. Our starting point in the study of the collapse of an A chain in a melt of B chains is thus the free energy

$$
\frac{F}{k T}=\frac{3}{2\left(1-c a^{3}\right)} \frac{N a^{2}}{R e}+\frac{N}{P c a^{3}}\left(1-c a^{3}\right) \log \left(1-c a^{3}\right)+\frac{N}{c a^{3}} U_{\mathrm{int}}(c)+\frac{3 R^{2}}{2 N a^{2}}
$$

or :

$$
\frac{F}{k T}=\frac{3}{2\left(1-c a^{3}\right)} \frac{N a^{2}}{R^{2}}+\frac{3}{2} \frac{R^{2}}{N a^{2}}+\frac{N}{P c a^{3}}\left(1-c a^{3}\right) \log \left(1-c a^{3}\right)+\frac{N}{c a^{3}} \frac{U_{\mathrm{int}}(c)}{k T} .
$$

We can now study the radius $R$ as a function of temperature in two limits :

i) When the temperature is very low i.e. $\tau \ll-1$.

ii) When the temperature is close to the compensation point i.e. $|\tau| \ll 1$.

However we will limit ourselves to a long chain among shorter chains. The completely collapsed radius of the $N$ chain is $N^{1 / 3} a$. We restrict ourselves to the limit where this radius is much larger than the Gaussian radius of the $P$ chains, i.e. $N^{1 / 3} / P^{1 / 2} \gg 1$.

i) Very low temperatures $|\tau| \gg 1 \tau$ negative. At very low temperatures, we expect the concentra- tion $c a^{3}$ to be close to 1 . We can introduce $\varepsilon=1-c a^{3}$. It can be checked $a$ posteriori that, at the equilibrium, $e$ is much smaller than the radius $R$. We thus use the free energy $\left(3^{\prime}\right)$. In the limit $\varepsilon \ll 1$, substituting the values of $e$ and $R$ as functions of $c$, and keeping only the binary attractions $k T \chi c(1-c)$ in $U_{\text {int }}$, the free energy can be written

$$
\frac{F}{k T}=\frac{3}{2 \varepsilon} \frac{N^{2 / 3} \cdot \tau^{1 / 2}}{P^{1 / 2}}-\frac{\varepsilon \tau N}{P} .
$$

This means that at very low temperatures the two dominant parts in the free energy are the binary attraction between monomers and the interfacial 
energy. Minimization of the free energy (5) gives the concentration inside the A chain :

$$
c a^{3}=1-\frac{1}{\tau^{1 / 3}}\left[\frac{P^{1 / 2}}{N^{1 / 3}}\right]^{2 / 3} .
$$

This should be the case for normal melts at room temperature when they are incompatible.

ii) Temperatures close to the compensation point $|\tau| \ll 1$. - At higher temperatures, for quasi-incompatible melts, we expect a much lower concentration. In this limit, the interfacial term is never dominant and one can describe the melt by the free energy $\left(4^{\prime}\right)$ in the limit of small concentrations. The free energy is then

$$
\begin{aligned}
\frac{F}{k T}=\frac{3}{2} \frac{R^{2}}{N a^{2}}+\frac{3}{2} \frac{N a^{2}}{R^{2}} & +\frac{N}{c} \frac{U_{\mathrm{int}}(c)}{k T}+ \\
& +\frac{N}{P c}\left(-c+\frac{c^{2}}{2}+\frac{c^{3}}{6}\right) .
\end{aligned}
$$

In that approximation, we do not limit ourselves to two-body interactions in $U_{\text {int }}(c)$. We make an expansion $U_{\text {int }}=k T \chi c(1-c)+A_{3} c^{3}$. We can rewrite the free energy (7) in terms of the concentration, alone, dropping all the constant terms.

$$
\begin{aligned}
\frac{F}{k T}=\frac{3}{2} \frac{1}{N^{1 / 3} c^{2 / 3}}+\frac{3}{2} c^{2 / 3} N^{1 / 3} & + \\
& +\frac{1}{2} \frac{\tau c N}{P}+N t c^{2}
\end{aligned}
$$

where $t$ is the third virial coefficient $t=\left(A_{3}+\frac{1}{6 P}\right)$ which we will assume to be positive. In the pure Flory theory $A_{3}=0$ and $t=1 / P$. However, for real polymers, there may exist a direct three-body interaction, in which case, the third virial is not screened, and $t$ is of order one. In the following, we distinguish between these two situations.

a) Screened third virial. - Minimization of the free energy with respect to $c$ gives implicitly the equilibrium concentration inside the A chain :

$$
-\tau=\frac{P}{N} \frac{N c}{P}+\frac{N^{1 / 3}}{c}-\frac{1}{N^{1 / 3} c^{5 / 3}} .
$$

In figure 1, we give a plot of the «temperature » as a function of concentration.

As in the case of polymers in a poor solvent, this curve is not monotonic. This result is obviously nonphysical, which is the reason why some authors talk about a coil-globule phase transition. However, this could be due to an inconsistency in the self-consistent field theory as proposed by Moore [6]. Anyhow there is a big jump in concentration and consequently the radius of the $A$ coil varies very sharply inside the $S$ region of the curve. We propose to characterize that

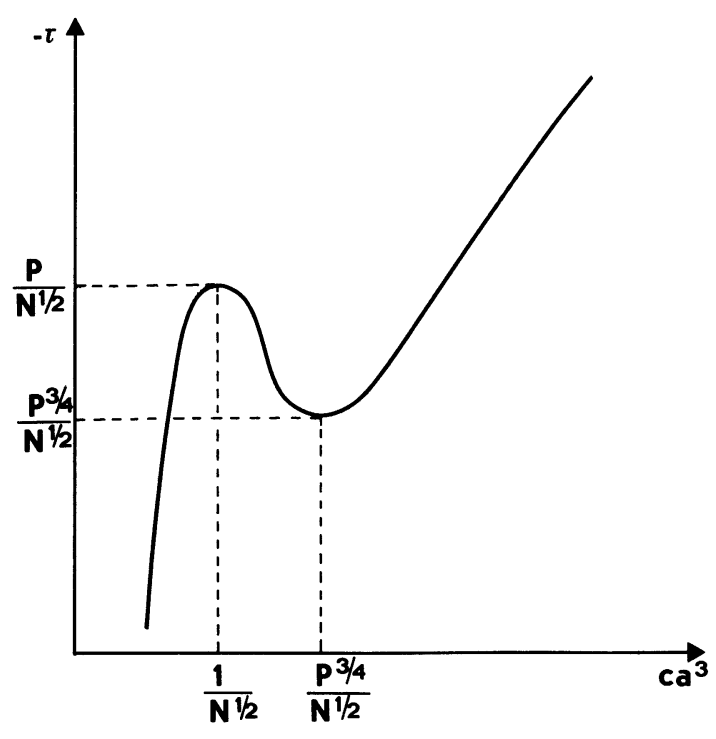

Fig. 1. - Temperature concentration relation at equilibrium.

steep variation by what we will call a « contraction point ", obtained by equating the free energy on the two branches of the curve $(-\tau, c)$ :

- On the left hand side

$$
c \sim \frac{1}{N^{1 / 2}} \text { and } \frac{F}{k T} \approx 1+\frac{N^{1 / 2} \tau}{P} .
$$

- On the right hand side $c \sim(-\tau)$ and

$$
\frac{F}{k T}=-\frac{N}{P} \tau^{2}+|\tau|^{2 / 3} N^{1 / 3}
$$

The temperature of the contraction point scales then as

$$
-\tau \sim \frac{P^{3 / 4}}{N^{1 / 2}}
$$

This means that in the non-monotonic region, the collapsed state is in fact more stable than the ideal state. However one can expect very strong hysteresis effects in the temperature region

$$
\frac{P^{3 / 4}}{N^{1 / 2}}<-\tau<\frac{P}{N^{1 / 2}}
$$

To sum up, we plot the radius as a function of concentration at all temperatures (Fig. 2).

b) Non-screened third virial coefficient. - The case of a free energy with a non-screened virial coefficient can be studied in the same way. The results look qualitatively the same but the hysteresis region is somewhat smaller. The contraction temperature is given by $(-\tau)=P / N^{1 / 2}$ (Fig. 3). For practical situations, the existence of a non-screened third virial coefficient seems more realistic. In such situations, the sharpness of the transition is much reduced. 


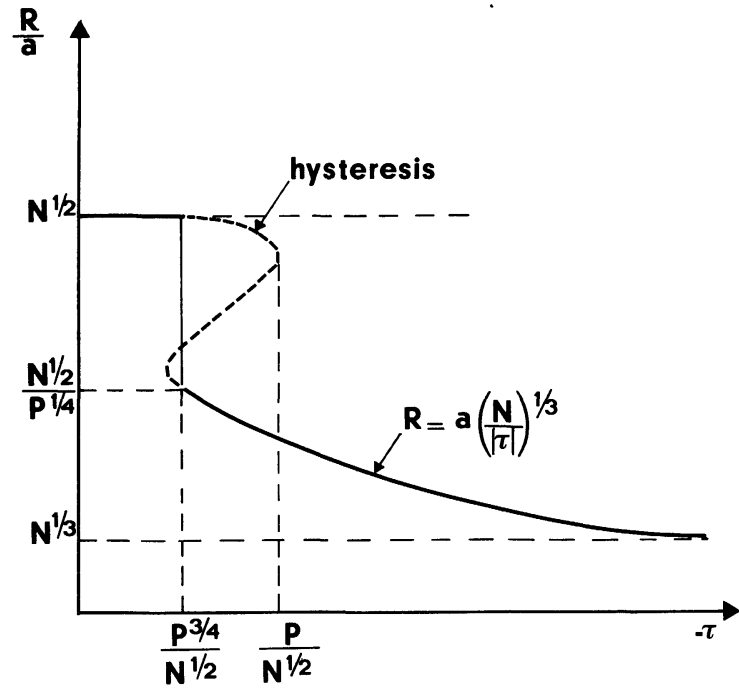

Fig. 2. - Variation of the radius of gyration with temperature (screened interactions).

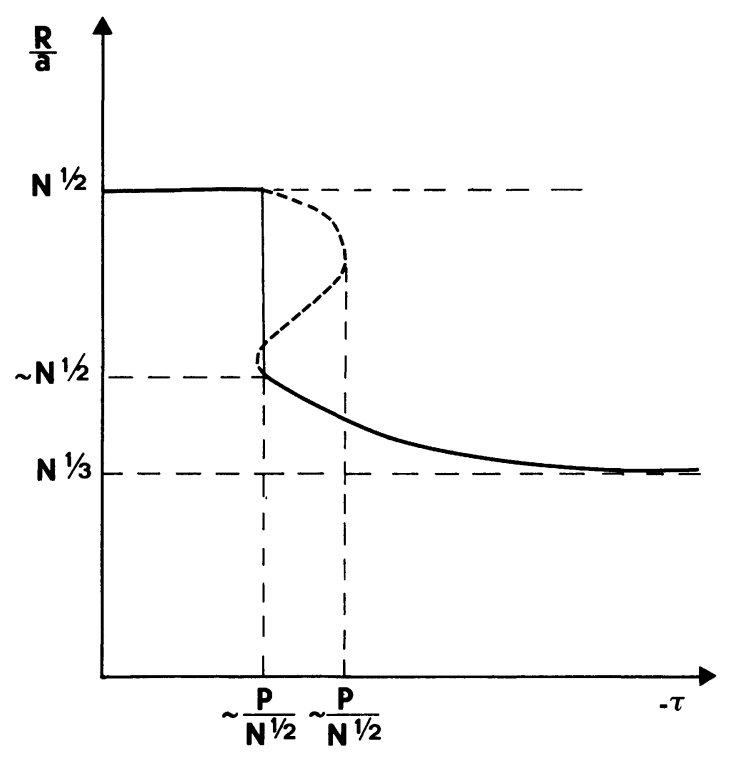

Fig. 3. - Variation of the radius of gyration with temperature (unscreened interactions).

3. Conclusion. - We have determined the temperature region inside which an $\mathrm{A}$ chain of. $\mathrm{V}$ monomers collapses in a melt of incompatible B chains of $P$ monomers $(P \ll N)$. If we compare this " coilglobule transition " with the usual collapse of a single polymer chain in a poor solvent, we find that the physics of the problem is very similar; however, there are two important differences :

- the $\theta$ region (i.e. the temperature domain where the $N$ chains are ideal is somewhat larger);

- the "transition" at the contraction point is much sharper, the non-monotonic variation of the radius occurs over a much wider temperature range. We do not know if there is actually a transition as indicated by our mean field arguments but at least this theory indicates a very narrow transition region. The jump in the radius of gyration at the contraction point can be much larger. If we ignore the third virial coefficient (as in the usual Flory theory for melts) we have a jump of the radius of gyration from $N^{1 / 2}$ to $N^{1 / 2} / P^{1 / 4}$. A deeper understanding of the problem can be given by comparing the structure of the single collapsed chain with the one of the dense phase at the same temperature in a binary melt of polymers A and $\mathrm{B}$.

Let us consider for instance the problem of a melt of concentration $\rho_{N}$ of long $N$ chains and $\rho_{P}=1-\rho_{N}$ of shorter $P$ chains $(P \ll N)$. Such a system shows a phase separation with a phase diagram given in figure 4 .

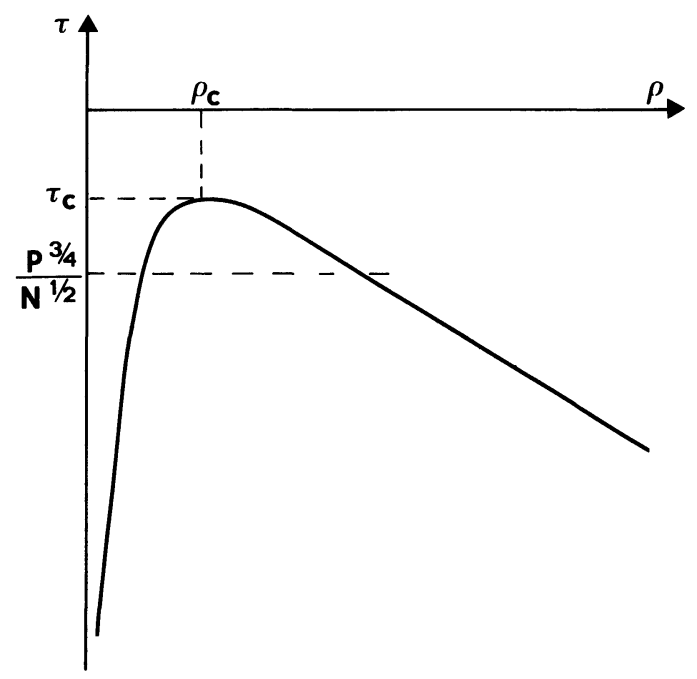

Fig. 4. - Flory's coexistence curve for a binary polymer mixture. The collapse occurs far below $\tau_{c}$ contrary to polymer poor solvent mixtures.

This phase diagram shows a critical point at $\tau_{\mathrm{c}}$ with $\tau_{\mathrm{c}} \approx \frac{P^{1 / 2}}{N^{1 / 2}}, \rho_{\mathrm{c}}=\frac{P^{1 / 2}}{N^{1 / 2}}$. Far below the critical temperature, there are very few correlations among the chains and the intrinsic properties of the chain are the same for a single chain $\left(\rho_{N}=0\right)$ as for chains in a dilute solution on the coexistence curve. On the coexistence curve, the $N$ chains tend to have an internal structure similar to the structure of the dense phase $\left(\rho_{N} \approx 1\right)$. That dense phase is characterized by a concentration $\rho_{\mathrm{d}}=|\tau|$ and a correlation length $\xi \approx P^{1 / 2} / C$. In the dilute phase the $N$ chains reproduce these properties, their radius scales like $\left[N / \rho_{\mathrm{d}}\right]^{1 / 3}$ and the thickness of the interfacial region is $\xi$. This gives back the results of section 3 . However, this picture breaks down at $R=\xi$. As usual, for $R<\xi$, the chain is ideal. It should be noted that contrary to the $P=1$ case, the collapse occurs far below the critical point.

Acknowledgments. - We are grateful to Professors P. G. de Gennes and P. Pincus for helpful discussions. 


\section{APPENDIX A}

Self-consistent field approximation. - In this appendix, the self-consistent approach of Moore [6] is generalized for the case of a melt, to derive the free energy of one A chain in a melt of B chains.

To describe the melt, we need two propagators; $G_{\mathrm{A}}(N, R)$ for the $\mathrm{A}$ chain and $G_{\mathrm{B}}(P, R)$ for the $\mathrm{B}$ chains [9]. Both of them are solutions of a Schrödinger type equation

$$
\begin{aligned}
& -\frac{\partial}{\partial N} G_{\mathrm{A}}(N, R)=-\frac{a^{2}}{6} \nabla^{2} G_{\mathrm{A}}+\frac{V_{\mathrm{A}}}{k T} G_{\mathrm{A}} \\
& -\frac{\partial}{\partial P} G_{\mathrm{B}}(P, R)=-\frac{a^{2}}{6} \nabla^{2} G_{\mathrm{B}}+\frac{V_{\mathrm{B}}}{k T} G_{\mathrm{B}}
\end{aligned}
$$

$V_{\mathrm{A}}$ and $V_{\mathrm{B}}$ being the potentials seen respectively by monomers A and B. $G_{\mathrm{A}}$ and $G_{\mathrm{B}}$ satisfy the boundary condition :

$$
\lim _{N \rightarrow 0} G_{\mathrm{A}}=\lim _{P \rightarrow 0} G_{\mathrm{B}}=\delta(r) .
$$

It is then convenient to expand $G_{i}(i=\mathrm{A}$ or B) in a series of eigenfunctions $U_{n}^{i}$ of the operator $H_{i}$ :

$$
H_{i}\left(U_{n}^{i}\right)=-\frac{a^{2}}{6} \nabla^{2} U_{n}^{i}+\frac{V_{i}}{k T} U_{n}^{i}=\varepsilon_{\mathrm{A}}^{i} U_{n}^{i} .
$$

The eigenfunctions are normalized to 1 so that

$$
\begin{gathered}
G_{\mathrm{A}}(N, r)=\sum U_{n}^{\mathrm{A}}(0) U_{n}^{\mathbf{A}}(r) \mathrm{e}^{-\varepsilon_{n}^{\mathbf{A}} N} \\
G_{\mathbf{B}}(P, r)=\sum U_{n}^{\mathbf{B}}(0) U_{n}^{\mathbf{B}}(r) \mathrm{e}^{-\varepsilon_{n}^{\mathbf{B} P}}
\end{gathered}
$$

In the melt of B chains, the local properties become $P$ independent and the sum giving $G_{\mathbf{B}}$ is dominated by the lowest eigenvalue [7]

$$
G_{\mathbf{B}}(P, r)=\mathrm{e}^{-\varepsilon_{0}^{\mathbf{B} P}} U_{0}^{\mathbf{B}}(0) U_{0}^{\mathbf{B}}(r)
$$

Following Moore, we also make a ground state dominance approximation for

$$
G_{\mathrm{A}}=G_{\mathrm{A}}(N, r)=\mathrm{e}^{-\frac{\mathbf{A}}{\varepsilon_{0} N}} U_{0}^{\mathrm{A}}(0) U_{0}^{\mathbf{A}}(r) .
$$

That approximation is valid as soon as $\left(\varepsilon_{0}^{\mathbf{A}}-\varepsilon_{1}^{\mathbf{A}}\right) N \gg 1$. With these approximations, it is well known $[6,7]$ that the concentration $C_{i}(r)$ of monomers $i$ is proportional to $\left[U_{0}^{i}(r)\right]^{2}$. We introduce new functions $\Psi_{\mathrm{A}}$ and $\Psi_{\mathrm{B}}$ proportional to $U_{0}^{\mathrm{A}}$ and $U_{0}^{\mathrm{B}}$ and such that

$$
\begin{aligned}
& C_{\mathrm{A}}=\Psi_{\mathrm{A}}^{2} \\
& C_{\mathrm{B}}=\Psi_{\mathrm{B}}^{2} .
\end{aligned}
$$

The fields $\Psi_{\mathrm{A}}$ and $\Psi_{\mathrm{B}}$ are then normalized by

$$
\int \Psi_{\mathrm{A}}^{2} \mathrm{~d}^{3} r=N \quad \int \Psi_{\mathrm{B}}^{2} \mathrm{~d}^{3} r=P \mathcal{C}
$$

where $\mathcal{N}$ is the number of $\mathrm{B}$ chains in the sample.
The next step amounts to making the theory selfconsistent by choosing $V_{\mathrm{A}}$ and $V_{\mathrm{B}}$. In a melt of $\mathrm{B}$ chains with one A chain, the Flory free energy per unit volume is given by

$$
F=\frac{k T}{P} C_{\mathrm{B}} \log C_{\mathrm{B}}+U_{\mathrm{int}}\left(C_{\mathrm{A}} C_{\mathrm{B}}\right)
$$

The first term represents the translational energy of the $\mathbf{B}$ chains, the second term, the interaction energy. In the usual Flory theory, $U_{\text {int }}=k T \chi C_{\mathrm{A}} C_{\mathrm{B}}$ but we can allow here a more general form :

$$
U=k T \chi C_{\mathrm{A}} C_{\mathrm{B}}+\sum_{i>3} A_{i} C_{\mathrm{A}}^{i}
$$

There is no translational term for the A chain in the free energy $A$ [8]. There is just one A chain and the average concentration $\bar{C}_{\mathrm{A}} / N$ of $\mathrm{A}$ chains is zero, which cancels the translational free energy of the A chain. The potential seen by the $i$ monomer $V_{i}$ can be taken to be :

$$
V_{i}=\frac{\mathrm{d}}{\mathrm{d} C_{i}} F\left(C_{\mathrm{A}} C_{\mathrm{B}}\right) .
$$

In the framework of the self-consistent field approximation, we are thus lead to a system of coupled differential equations for the fields $\Psi_{\mathrm{A}}$ and $\Psi_{\mathrm{B}}$ :

$$
\begin{aligned}
& \varepsilon_{0}^{\mathrm{A}} \Psi_{\mathrm{A}}=-\frac{a^{2}}{6} \nabla^{2} \Psi_{\mathrm{A}}+\frac{\partial F}{\partial C_{\mathrm{A}}} \Psi_{\mathrm{A}} \\
& \varepsilon_{0}^{\mathrm{B}} \Psi_{\mathrm{B}}=-\frac{a^{2}}{6} \nabla^{2} \Psi_{\mathrm{B}}+\frac{\partial F}{\partial C_{\mathrm{B}}} \Psi_{\mathrm{B}}
\end{aligned}
$$

Equations (A.10) can be derived by a variational principle from the functional

$$
\begin{aligned}
F=\int \mathrm{d}^{3} r \frac{k T a^{2}}{6} \times \\
\quad \times\left[\left(\nabla \Psi_{\mathrm{A}}\right)^{2}+\left(\nabla \Psi_{\mathrm{B}}\right)^{2}+F\left(C_{\mathrm{A}}, C_{\mathrm{B}}\right)\right] .
\end{aligned}
$$

The two constants $\varepsilon_{0}^{\mathbf{A}}$ and $\varepsilon_{0}^{\mathbf{B}}$ are then introduced as Lagrange multipliers insuring that the normalization conditions (A.7) are respected. Physically, we may

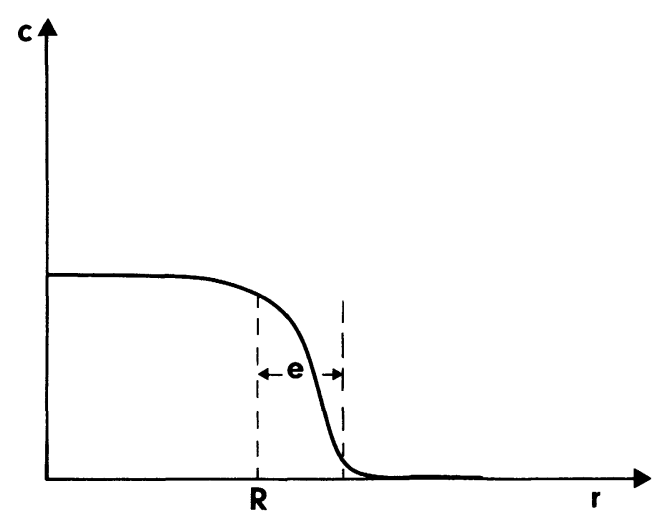

Fig. A1. - Concentration profile inside an A polymer coil. 
think of $F$ as the free energy of our A chain immersed in a melt of $B$ chains.

We can rewrite the free energy $F$ solely in terms of the local concentration of monomers A $C=C_{\mathrm{A}}$. The local incompressibility imposes $a^{3} C_{\mathrm{B}}=1-\mathrm{Ca}^{3}$ and the free energy of our A chain is

$$
F=\int \mathrm{d}^{3} r \frac{a^{2}}{24}\left(\frac{1}{C a^{3}}+\frac{1}{1-C a^{3}}\right)(\nabla C)^{2}+F(C)
$$

Such a free energy introduces naturally a characteristic length $e$

$$
e^{2}=\frac{a^{2}}{12}\left(\frac{1}{C a^{3}}+\frac{1}{1-C a^{3}}\right) \frac{P}{|\tau|}
$$

$\tau$ being related to the Flory's interaction parameter $\chi$ by

$$
\tau=1-2 P_{\chi} \text {. }
$$

As in Moore's paper, the self-consistent approach is valid as soon as the characteristic length $e$ is smaller than the radius $R$ of the A coil. When this is true, the concentration profile of the A chain has the shape given in figure $\mathrm{A} 1$, and we can interpret the gradient terms of equation (A.12) as an interfacial energy.

\section{References}

[1] Flory, P. J., Principles of Polymer Chemistry (Cornell University Press) 1953.

[2] De Gennes, P. G., Scaling Concepts in polymer Physics (Cornell University Press) 1979.

[3] Manson, J., Sperline, M., Polymer Blends and Composites (London, Heyden) 1976.

[4] See the reviews on the collapse of a Polymer chain : LIFsChitz, I. M., Grosberg, A., Khokhlov, A., Rev. Mod. Phys. 50 (1978) 685.

Brochard, F., Frisch, H. L., Williams, C., to be published.

[5] Cantor, R., Accepted for publication in Macromolecules.
[6] Moore, M. A., J. Phys. A. Math. Gen. 10 (1977) 305.

[7] Joanny, J. F., Leibler, L., De Gennes, P. G., J. Polvm. Sci. Polym. Phys. Ed. 17 (1979) 1073.

[8] De Gennes, P. G., J. Physique Lett. 36 (1975) L-55 ; J. Physique Lett. 39 (1978) L-299.

[9] Helfand, E., J. Chem. Phys. 62 (1975) 999 ; Macromolecules 9 (1976) 879.

[10] Sun, S. T., Nishio, I., Swislow, G., Tanaka, T., J. Chem. Phys. $73(1980) 5971$.

[11] Bauer, D. R., Ullman, R., Macromolecules 13 (1980) 392. 